\title{
Targeting effect of PEGylated liposomes modified with the Arg-Gly-Asp sequence on gastric cancer
}

\author{
JIE DING, MIN FENG, FENG WANG, HAO WANG and WENXIAN GUAN \\ Department of General Surgery, Nanjing Drum Tower Hospital, The Affiliated Hospital of Nanjing University \\ Medical School, Nanjing, Jiangsu 210008, P.R. China
}

Received March 19, 2015; Accepted June 26, 2015

DOI: 10.3892/or.2015.4142

\begin{abstract}
Previous studies have demonstrated that the $\alpha_{5} \beta_{1}$ integrin-mediated interaction with fibronectin (FN) occurs through the Arg-Gly-Asp (RGD) cell-binding sequence in repeat $\mathrm{III}_{10}$. Indocyanine green (ICG) is a near-infrared (NIR) optical dye that has been approved by the US Food and Drug Administration. In the present study, we developed an RGD-modified PEGylated liposome-encapsulated ICG (RGD-PLS-ICG) system mediated by integrin. RGD was conjugated covalently to the distal end of DSPE- $\mathrm{PEG}_{2000}-\mathrm{NH}_{2}$ lipid by amide binding. The characteristics and stability of the prepared liposomes were assessed. In vitro, SGC7901 cells with high expression of integrin $\alpha_{5} \beta_{1}$ were selected by polymerase chain reaction (PCR) and western blotting. To confirm the targeting efficacies to gastric cancer, coumarin- 6 was encapsulated as a fluorescent probe for in vitro study, and the targeting effect of RGD was detected by flow cytometry and confocal microscopy. In vivo, the bio distribution of RGD-PLS-ICG was studied by an in vivo imaging system in the tumor model. RGD-PLS-ICG and PLS-ICG had a higher UV absorbance spectrum and stability than free-ICG. Confocal microscopy and flow cytometry demonstrated that RGD-PLS-encapsulated coumarin-6 was efficiently associated with the SGC7901 cells, while limited interaction was found for the other groups. Moreover, the in vivo imaging of the liposomes indicated that RGD-PLS-ICG achieved more accumulation in the tumor tissues when compared with PLS-ICG. The significant in vitro and in vivo results suggest that RGD-PLS-ICG may be a promising fluorescent dye delivery system for targeting gastric cancer cell overexpression of integrin.
\end{abstract}

Correspondence to: Dr Wenxian Guan, Department of General Surgery, Nanjing Drum Tower Hospital, The Affiliated Hospital of Nanjing University Medical School, Nanjing, Jiangsu 210008, P.R. China

E-mail: ddingnjuer@sina.com

Key words: gastric cancer, liposomes, RGD, targeting, indocyanine green

\section{Introduction}

Gastric cancer is the fourth most common cancer and the second leading cause of death from malignant disease worldwide, with especially high mortality rates in East, South and Central Asia; in Central and Eastern Europe; and in South America (1). In D1 dissection, the stomach (total or distal) plus the perigastric lymph nodes are removed; and in D2 dissection, additional removal of the nodes along the left gastric area, common hepatic splenic zone and left hepatoduodenal artery is performed (2). The 3rd edition of 'The Gastric Cancer Treatment Guidelines' in Japan, defined D2 radical surgery as the standard operation. In recent years, D2 radical surgery has also been gradually accepted by European and American doctors. The United States National Comprehensive Cancer Network and the European Society for Medical Oncology have recommended D2 radical surgery to improve the 5-year survival rate of gastric cancer patients $(3,4)$. However, follow-up study conducted over 15 years suggested that D2 operation is associated with lower locoregional recurrence and gastric-cancer-related death rates, yet had significantly higher postoperative mortality, morbidity and reoperation rates than D1 surgery (5). Obviously, a standardized operation may lead to an expansion in the scope of relative lymphadenectomy in some patients and increase unnecessary damage and risk. Therefore, the accurate resection of tumors, even accurate lymphadenectomy, is urgent and necessary.

Integrin is a major family of cell surface receptors. It serves as the bridge for cell-cell and cell-extracellular matrix (ECM). Integrin $\alpha_{5} \beta_{1}$, is one of the important members of the integrin family and is a major receptor of fibronectin (FN). Previous research has shown that integrin $\alpha_{5} \beta_{1}$ identifies and combines with the Arg-Gly-Asp (RGD) sequence, an RGD sequence that is located in $\mathrm{III}_{10}$ of $\mathrm{FN}$ and is the site of cell attachment via $\alpha_{5} \beta_{1}$ integrin on the cell surface (6-9). Integrin $\alpha_{5} \beta_{1}$ plays an important role in tumor growth, invasion and metastasis (10-12). In recent years, research suggests that expression of integrin is associated with the differentiation and metastasis of gastric cancer (13), and integrin $\alpha_{5} \beta_{1}$ may be an independent prognostic factor for gastric cancer patients (14). Therefore, artificial synthetic RGD, or an agent containing the RGD sequence has been used as a ligand to target gastric cancer $(15,16)$.

Indocyanine green (ICG) was approved by the FDA for use as a clinical near-infrared imaging agent, which has high safety, less adverse reactions, and a high signal-to-noise ratio 
in living tissue. It has been used for gastric cancer sentinel lymph node (SLN) imaging $(17,18)$. However, the intravenous administration of ICG has a short half-life; for this reason, its intravenous ICG study as a gastric cancer tracer has not been carried out. Thus, it is necessary to search for new strategies for prolong the half-live of ICG in vivo.

Liposomes were first described by the British hematologist A.D. Bangham (19). Liposomes are artificially prepared spherical vesicles composed of a lamellar phase lipid bilayer. Liposomes have various advantages, such as biocompatibility and no obvious toxicity or immunogenicity. Liposomes have been used as an antitumor drug delivery carrier for the treatment of tumors, as previously reported $(20,21)$. However, liposomes are easily utilized by the reticuloendothelial system (RES) and gather in organs, such as the liver and spleen. In order to avoid or reduce the uptake of RES, researchers have prepared liposomes that are modified with polyethylene glycol (PEG), which effectively prolongs their circulation time. In recent years, researchers have used PEG-modified liposomes in combination with targeted ligands (RGD, trastuzumab) to treat tumors in animal models $(22,23)$; and PEGylated liposome (PLS)-encapsulated ICG has reportedly been used for the identification of increased vascular permeability in arthritis disease models (28) and lymphatic function imaging (24,25). However, previous research has not reported the use of liposome-encapsulated ICG to trace tumors.

In the present study, preparation of PLS-ICG was carried out using the lipid thin-film hydration/extrusion method. RGD was then added to the end of the PEG chain by the amidation reaction. The prepared liposomes were used in a tracing study of gastric cancer in a xenograft nude mouse model. The liposomes effectively avoided uptake by the RES and exhibited prolonged circulation time in vivo; furthermore, they are passive in targeting tumor tissue by enhanced permeability and retention effect (EPR) (26), are active in targeting tumors through RGD, and increase the ICG accumulation in tumors. The results of the present study may be able to help surgeons toward greater accuracy in the resection of tumors under the guidance of a tracer.

\section{Materials and methods}

Preparation of liposomes. Preparation of the liposomes was performed using the lipid thin-film hydration/extrusion method (27). In brief, L- $\alpha$-phosphatidylcholine (PC; purity $>98 \%$; Aladdin Industrial Corporation, City of Industry, CA, USA), cholesterol (CHOL; Sigma-Aldrich, St. Louis, MO, USA) and 1,2-distearoyl-sn-glycero-3-phosphoethanolamine$\mathrm{N}$-[methoxy(polyethylene glycol)-2000] (DSPE-PEG ${ }_{2000}$ ), 1,2-distearoyl-sn-glycero-3-phosphoethanolamine-N-[car boxy(polyethylene glycol)-2000] (ammonium salt) (DSPE$\mathrm{PEG}_{2000}-\mathrm{NH}_{2}$; Avanti Polar Lipids, Inc., Alabaster, AL, USA) in a molar ratio of 2:1:0.08:0.02 were dissolved in chloroform/ methanol (2:1). The organic solvent was evaporated under nitrogen, and the lipid film was placed under vacuum overnight. The film was completely hydrated using a glucose buffer solution $(5 \%, \mathrm{w} / \mathrm{v})$ with dissolved ICG $(15 \mathrm{mmol})$. The ICG-containing mixture was freeze-thawed 8 times and extruded 10 times through a polycarbonate film with a pore size of $100 \mathrm{~nm}$ using the mini-lipid extruder (Morgan Machine
Co. Inc., USA) to obtain small unilamellar vesicles. Free-ICG was removed by size exclusion chromatography on a PD Midi-Trap G-25 column (GE Healthcare, Uppsala, Sweden) using glucose buffer as the eluent.

Coumarin-6-loaded liposomes were prepared using the same procedures as previously mentioned, and the liposome suspension was eluted using a Sephadex LH-20 column (GE Healthcare) to remove free coumarin-6.

Attachment of RGD (Shanghai HD Biosciences Co., Shanghai, China) to the liposomes was based on free carboxyl group of RGD and DSPE-PEG $2000-\mathrm{NH}_{2}$ from liposomes with 4-(4,6-dimethoxy-1,3,5-triazin-2-yl)-4-methylmorpholinium chloride (DMTMM; Sigma-Aldrich) as catalysts (28). Briefly, the liposomes of the above preparation were incubated with RGD and DMTMM (DSPE-PEG $2000-\mathrm{NH}_{2}:$ RGD:DMTMM=1:1:1, molar ratio), and then the mixture was slightly stirred for $1 \mathrm{~h}$ at room temperature. The excess DMTMM and RGD were removed using a Sephadex G-50 Mini-Column (Piscataway, NJ, USA).

Characteristics of the liposomes. The particle size and size distribution of the liposomes were measured at $25^{\circ} \mathrm{C}$ by photon correlation spectroscopy at a scattering angle of $90^{\circ}$ using a Zetasizer Nano ZS90 (Malvern Instruments, Worcestershire, UK). Each measurement was repeated 3 times for each sample. The morphology of the liposomes was observed using an H-7650 transmission electron microscope (TEM; Hitachi Ltd., Tokyo, Japan) with $2 \%$ phosphotungstic acid stain.

Stability of the liposomes. The UV absorbance spectra were obtained using an Ultrospec 2100 pro UV/Visible Spectrophotometer (GE Healthcare Life Sciences) at wavelengths from 600 to $900 \mathrm{~nm}$. The stability of the liposome-encapsulated ICG was monitored by a dialysis method measuring the free-ICG concentration as an indicator of liposome degradation. The dialysis system included solvent with $50 \%$ fetal bovine serum (FBS) as a condition of blood, loaded ICG liposomes and $50 \%$ FBS in the membrane (MWCO 500; Spectrum) inlet and only $50 \%$ FBS in the membrane outlet as previously described (29).

Cell culture. Human gastric cancer cell lines SGC7901, BGC823 and MGC803, and human mucosa endothelial cell line GES1 were purchased from the Shanghai Institute of Cell Biology (Chinese Academy of Sciences, Shanghai, China). Cells were cultured in Dulbecco's modified Eagle's medium (DMEM) supplemented with 10\% FBS, $100 \mathrm{U} / \mathrm{ml}$ penicillin and $100 \mu \mathrm{g} / \mathrm{ml}$ streptomycin (all from Gibco, Gaithersburg, $\mathrm{MD}, \mathrm{USA})$ at $37^{\circ} \mathrm{C}$ in a humidified atmosphere with $5 \% \mathrm{CO}_{2}$.

Quantitative real-time reverse transcription-polymerase chain reaction ( $q R T-P C R$ ). Total RNA was isolated from each cell line with the RNApure tissue kit following the manufacturer's instructions. The RNA samples were treated with DNase I (both from ComWin Biotech Co., Ltd., Beijing, China) to exclude contamination with traces of genomic DNA. The amount of RNA isolated was quantified with a NanoDrop 200 spectrophotometer (Thermo Scientific, Wilmington, DE, USA) and $1 \mu \mathrm{g}$ of purified total RNA was reversed transcription using PrimeScript RT reagent kit with gDNA Eraser 
(Takara, Dalian, China) in a Thermal Cycler Dice Real-Time System. Revere transcription reaction was performed at $37^{\circ} \mathrm{C}$ for $15 \mathrm{~min}$, followed by $85^{\circ} \mathrm{C}$ for $15 \mathrm{sec}$ and cooled at $4^{\circ} \mathrm{C}$.

qPCR analysis was performed in accordance with the manufacturer's instructions using SYBR ${ }^{\circledR}$ Premix Ex Taq ${ }^{\mathrm{TM}}$ II (Tli RNaseH Plus) (Takara). Two microliters of reverse transcription reaction was used for a total $20 \mu \mathrm{l}$ quantitative PCR reactions in Applied Biosystems 7500 Real-Time PCR System (Life Technologies, Carlsbad, CA, USA). The parameters were as follows: stage 1, pre-degeneration, at $95^{\circ} \mathrm{C}$ for $30 \mathrm{sec}$; stage 2 , counting for 40 cycles, at $95^{\circ} \mathrm{C}$ for $5 \mathrm{sec}$, and $60^{\circ} \mathrm{C}$ for $34 \mathrm{sec}$; stage 3 , dissociation, at $95^{\circ} \mathrm{C}$ for $15 \mathrm{sec}, 60^{\circ} \mathrm{C}$ for $1 \mathrm{~min}$, at $95^{\circ} \mathrm{C}$ for $15 \mathrm{sec}$. In comparison to the relative gene expression between the experimental and control groups, the $2^{-\Delta \Delta \mathrm{Ct}}$ values were calculated (30), where $\Delta \Delta \mathrm{Ct}=(\Delta \mathrm{Ct}$ value of the experimental group - $\Delta \mathrm{Ct}$ value of the control group) and $\Delta \mathrm{Ct}=(\mathrm{Ct}$ value of a selected gene $-\mathrm{Ct}$ value of $\beta$-actin $)$. The following sets of primers were used in the PCR amplification: $\beta$-actin (forward, 5'-GCGGGAAATCGTGCGTGAC-3' and reverse, 5'-CAGGAAGGAAGGCTGGAAGAGTG-3'); $\alpha 5$ (forward, 5'-GGATACTCTGTGGCTGTTGGTGAA-3' and reverse, 5'-GGATGGTGACATAGCCGTAAGTGA-3'); $\beta 1$ (forward, 5'-ACTATCCCATTGACCTCTACTACCT-3' and reverse, 5'-GTAATCCTCCTCATTTCATTCATCA-3').

Western blot analysis. Whole cell lysates were prepared as previously described (31). Total protein concentrations were determined by a Bicinchoninic acid protein assay kit (KeyGen, Jiangsu, China). Equal amounts $(30 \mu \mathrm{g})$ of protein extracted from the cultured cells were run on $10 \%$ SDS-PAGE, followed by electro-transferring to polyvinylidene fluoride membranes (Millipore, Bedford, MA, USA). The membranes were blocked with 5\% skim milk powder for $2 \mathrm{~h}$. Then, the membranes were incubated overnight at $4{ }^{\circ} \mathrm{C}$ with the primary antibodies anti-integrin $\alpha_{5}(1: 400)$, anti-integrin $\beta_{1}$ (1:400) (both from Abcam, USA), then with horseradish peroxidase-conjugated secondary antibody goat anti-rabbit IgG (1:2,000; ZSGB-Bio, Beijing, China) for $2 \mathrm{~h}$ at room temperature. The membranes were visualized by chemiluminescence (Millipore) and exposed with an automatic exposure machine (Bio-Rad, USA) in a dark room, and then Quantity One software was used for gray-scale analysis.

Flow cytometric analysis. The cell binding and internalization of the various coumarin-6-loaded liposomes were analyzed by flow cytometry (32). GES1, SGC7901, BGC823 and MGC803 cells were seeded onto 6 -well plates with about $6 \times 10^{5}$ cells/ well and cultured for $24 \mathrm{~h}$. Then, the cells were incubated for $1 \mathrm{~h}$ at $37^{\circ} \mathrm{C}$ with coumarin-6-loaded PLS and RGD-PLS in serum-free medium, in which the final concentration of coumarin- 6 was $2 \mu \mathrm{g} / \mathrm{ml}$. The free culture medium was applied as the blank control. After incubation, the cells were washed 3 times with cold phosphate-buffered saline (PBS; $\mathrm{pH}$ 7.4) and the cells underwent digestion with trypsin. Next, the cells were collected by centrifugation; resuspended in $500 \mu 1$ of PBS, followed by centrifugation and resuspension was repeated 3 times. The cellular uptake of the fluorescence was measured on a BD FACSCanto II flow cytometer (BD Biosciences, USA) equipped with an argon ion air cooled laser (488 nm). Approximately 10,000 events were counted for each sample.
Confocalmicroscopic analysis. The binding and internalization of the liposomes were also examined by laser confocal fluorescence microscopy (32). Aliquots of 5x10 4 SGC7901 and GES1 cells were seeded in 35-mm dishes with a glass coverslip at the bottom. After a 24-h proliferation, the cells were incubated with coumarin-6-loaded PLS and RGD-PLS in serum-free medium for $1 \mathrm{~h}$ at $37^{\circ} \mathrm{C}$. The final concentration of coumarin- 6 was $2 \mu \mathrm{g} / \mathrm{ml}$. After incubation, the medium was removed and the cells were washed 5 times with cold PBS. Next, the cells were fixed with $4 \%$ of p-formaldehyde (PFA) for $15 \mathrm{~min}$, followed by cell nuclear staining with Hoechst 33342 (Molecular Probes, Inc., Eugene, OR, USA) for $10 \mathrm{~min}$, and then washed 5 times with PBS for confocal microscopy analysis under oil mirror in magnification of 63 folds. The fluorescence images were analyzed with a laser confocal fluorescence microscope (LSCM; Zeiss LSM 710, Germany).

In vivo imaging in the gastric cancer animal model. Male BALB/C nude mice (18-20 g) were obtained from SLAC Laboratory Animal Co., Ltd. (Shanghai, China). All animals were treated according to the guidelines for the use of experimental animals and approved by the Institutional Animal Care and Research Advisory Committee at Nanjing University (Nanjing, China). ICG-loaded liposomes were used to investigate the tumor targeting efficacy in a heterotopic transplantation tumor model. Briefly, SGC7901 cells (5x10 6 cells/ mouse) were subcutaneously transplanted into the armpit of nude mice. Tumor volume was measured by a Vernier caliper and calculated as [length $\mathrm{x}$ (width) $\left.{ }^{2}\right] / 2$. When the tumor volume reached a size of $\sim 150-200 \mathrm{~mm}^{3}$, the mice were injected with PLS, ICG loaded PLS and ICG loaded RGD-PLS at a dose of $40 \mathrm{mg} \mathrm{ICG} / \mathrm{kg}$ body weight via tail vein, respectively. Animals were anesthetized with oxygen/air mixture containing $2 \%$ isoflurane. Fluorescence of the injected ICG was visualized using the Maestro EX in vivo fluorescence imaging system (CRi, Woburn, MA, USA). The imaging parameters were as follows: $\lambda$ ex $=735 \mathrm{~nm}, \lambda \mathrm{em}=840 \mathrm{~nm}$, exposure time $=1-5 \mathrm{sec}$, $\mathrm{f} / \mathrm{stop}=2$, medium binning, field of view $=6.6 \times 6.6 \mathrm{~cm}^{2}$. The mouse scans were carried out at 1, 2, 4, 8, 16, 24, 48, 72, 96 and $120 \mathrm{~h}$ post-injection. Thereafter, the tumor-bearing mice were sacrificed and the tumors, liver, spleen, kidney and stomach were harvested for isolated organ imaging.

Statistical analysis. Data are presented as mean \pm SD. For statistical analysis between two groups, the Student's t-test for independent means was used. A value of $\mathrm{P}<0.05$ was considered as statistically significant, and a P-value $<0.01$ was considered as very significant. Statistical analysis was performed using SPSS 19.0 software.

\section{Results}

Synthesis and characterization of the liposomes. The physical properties of the liposomes are shown in Table I. The mean size of the coumarin- 6 encapsulated PLS was $\sim 112 \mathrm{~nm}$ with a particle size distribution index (PDI) of 0.056 as measured by dynamic light scattering (DLS) method. The ICG-loaded liposomes were $\sim 106 \mathrm{~nm}$, with a PDI of 0.093 . Both of the liposomes were slightly increased following conjugation with RGD, yet there was no statistical significance and the PDI also 
Table I. Characteristics of coumarin-6 and ICG-loaded liposomes.

\begin{tabular}{llcc}
\hline Formulation & $\begin{array}{c}\text { Fluorescence } \\
\text { probe }\end{array}$ & $\begin{array}{c}\text { Particle size } \\
(\mathrm{nm})\end{array}$ & \multicolumn{1}{c}{ PDI } \\
\hline PLS & Coumarin-6 & $112.7 \pm 1.1$ & $0.056 \pm 0.014$ \\
RGD-PLS & Coumarin-6 & $119.6 \pm 2.4$ & $0.077 \pm 0.022$ \\
PLS & ICG & $106.4 \pm 2.2$ & $0.093 \pm 0.041$ \\
RGD-PLS & ICG & $117.5 \pm 2.1$ & $0.070 \pm 0.014$ \\
\hline
\end{tabular}

Data represent the mean \pm SD $(n=3)$. ICG, indocyanine green; PDI, particle size distribution index; PLS, PEGylated liposomes.

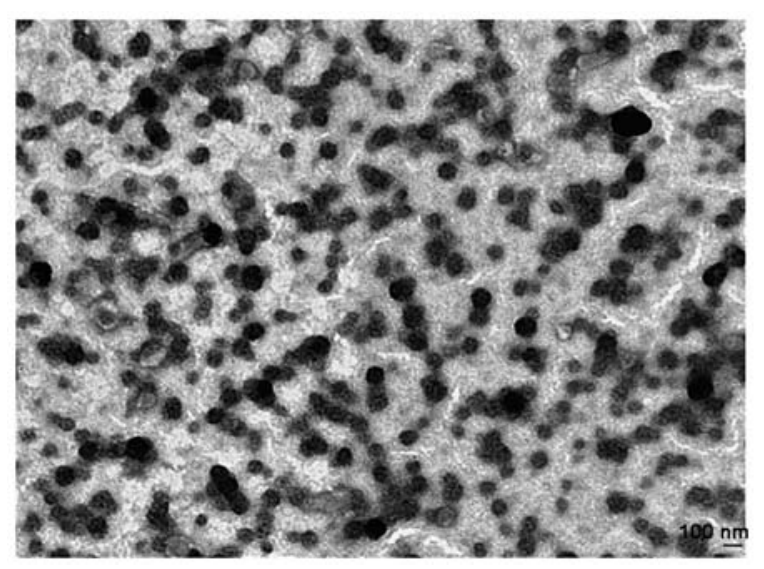

Figure 1. Morphology of the RGD-PLS-ICG was evaluated using TEM with $2 \%$ phosphotungstic acid stain. Scale bar, $100 \mathrm{~nm}$; original magnification, x50,000. RGD-PLS-ICG, RGD modified PEGylated liposome-encapsulated ICG; TEM, transmission electron microscopy.

did not show significant change. TEM (Fig. 1) indicated that the morphology of the liposomes were approximate circular and the particle size was similar. The results were consistent with the DLS.

Spectral properties and stability of the liposomes. Fig. 2 shows the absorption (Fig. 2A) spectra of free-ICG dissolved in a 50\% FBS solution compared with the spectra of LP-ICG and RGD-LP-ICG. The marked red shift $(23 \mathrm{~nm})$ in the absorption spectrum of the ICG-loaded liposomes confirmed the affinity of ICG for the lipid bilayers $(33,34)$. The seemingly minor shifts toward longer wavelengths resulted in a marked decrease in the in vivo background signal during detection, leading to an improved signal-to-noise ratio in vivo (25).

The stability of PLS-ICG and RGD-PLS-ICG was determined using the dialysis method to measure the free-ICG concentration as an indicator of liposome degradation. As illustrated in Fig. 2, the UV absorbance and ICG concentrations of PLS-ICG and RGD-PLS-ICG were higher than the free-ICG, and the ICG concentrations of PLS-ICG and RGD-PLS-ICG were slightly decreased over time, while the free-ICG was obviously decreased.

Integrin expression in the cell lines. Integrin $\alpha_{5} \beta_{1}$ was previously reported in gastric cancer $(13,35)$ and prostate
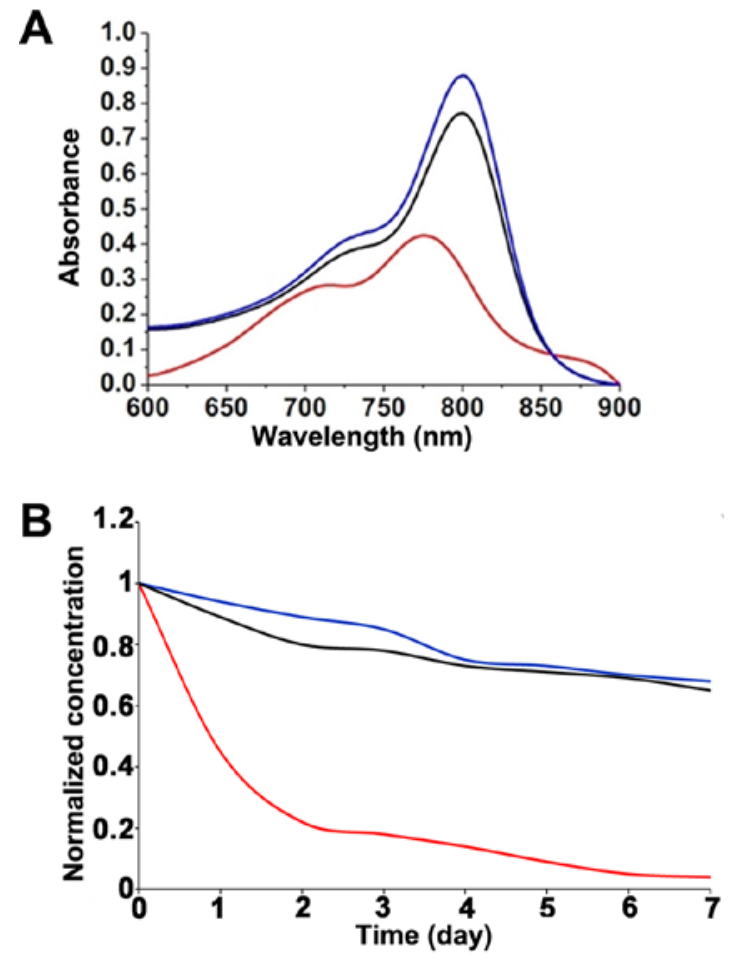

Figure 2. (A) UV absorbance spectra and (B) stability of ICG (red line), PLS-ICG (blue line) and RGD-PLS-ICG (black line) in the solution of $50 \% \mathrm{FBS}$ at $37^{\circ} \mathrm{C}$. ICG, indocyanine green; RGD-PLS-ICG, RGD modified PEGylated liposome-encapsulated ICG; FBS, fetal bovine serum.

cancer (36), and glioma (37), and those that have been revealed as FN receptors were explored. Fig. 3 shows the mRNA relative expression levels of integrin $\alpha_{5}$ and $\beta_{1}$ in the SGC7901, BGC823, MGC803 and GES1 cell lines. We found that the expression of $\alpha_{5}$ was higher in the SGC7901, BGC823 and MGC803 cells than that in the GES1 cells (Fig. 3A). $\alpha_{5}$ showed the highest level in the SGC7901 cells, while the expression level of $\alpha_{5}$ was higher in the BGC823 than that in the MGC803 cells. For $\beta_{1}$, the results revealed that $\beta_{1}$ had the highest expression level in the SGC7901 cells when compared with that in the GES1 cells, whereas MGC803 exhibited low expression. The expression level of $\beta_{1}$ exhibited no significant difference between the GES1 and BGC823 cells (Fig. 3B). As shown in Fig. 4, we also confirmed that the protein expression of integrin $\alpha_{5}$ and $\beta_{1}$ was higher in the SGC7901 cells than that in the BGC823, MGC803 and GES1 cell lines by western blotting. These results were consistent with PCR.

A certain type of $\alpha$-subunit integrin can be paired with a specific type of $\beta$-subunit; and it has been shown that integrin $\alpha_{5}$ binds only to $\beta_{1}$ (38). For integrin $\alpha_{5} \beta_{1}$, the expression of $\alpha_{5}$ determines the maximal levels of integrin $\alpha_{5} \beta_{1}$. Hence, we speculated that the expression of integrin $\alpha_{5} \beta_{1}$ was found in all cell lines, and SGC7901 cells showed the highest expression level when compared with that in the other cell lines.

Flow cytometric analysis of cell binding and cellular uptake. Flow cytometric analysis was performed to quantify the cell binding and cellular uptake. First, the uptake of RGD-PLS by SGC7901, BGC823, MGC803 and GES1 cells was compared to PLS, which chosen as the negative control. As shown in 
A

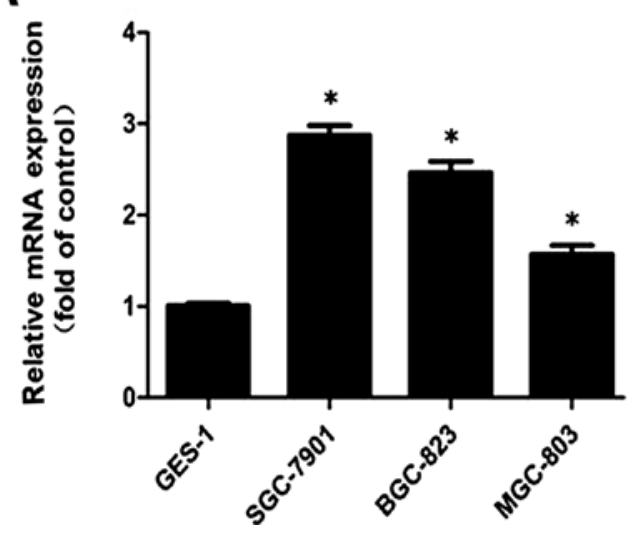

B

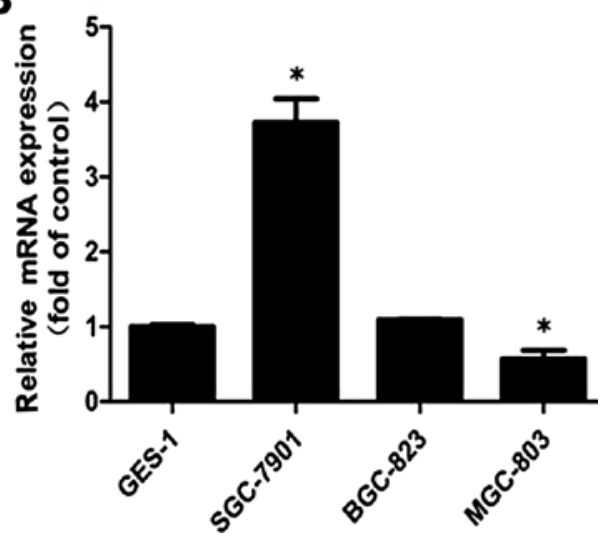

Figure 3. Gene expression of (A) integrin $\alpha_{5}$ and (B) $\beta_{1}$ subunits in human GES1, SGC7901, BGC823 and MGC803 cell lines. Data are expressed as relative gene expression after normalization against endogenous reference gene $\beta$-actin. ${ }^{*} \mathrm{P}<0.05$ (compared with GES1 cells).
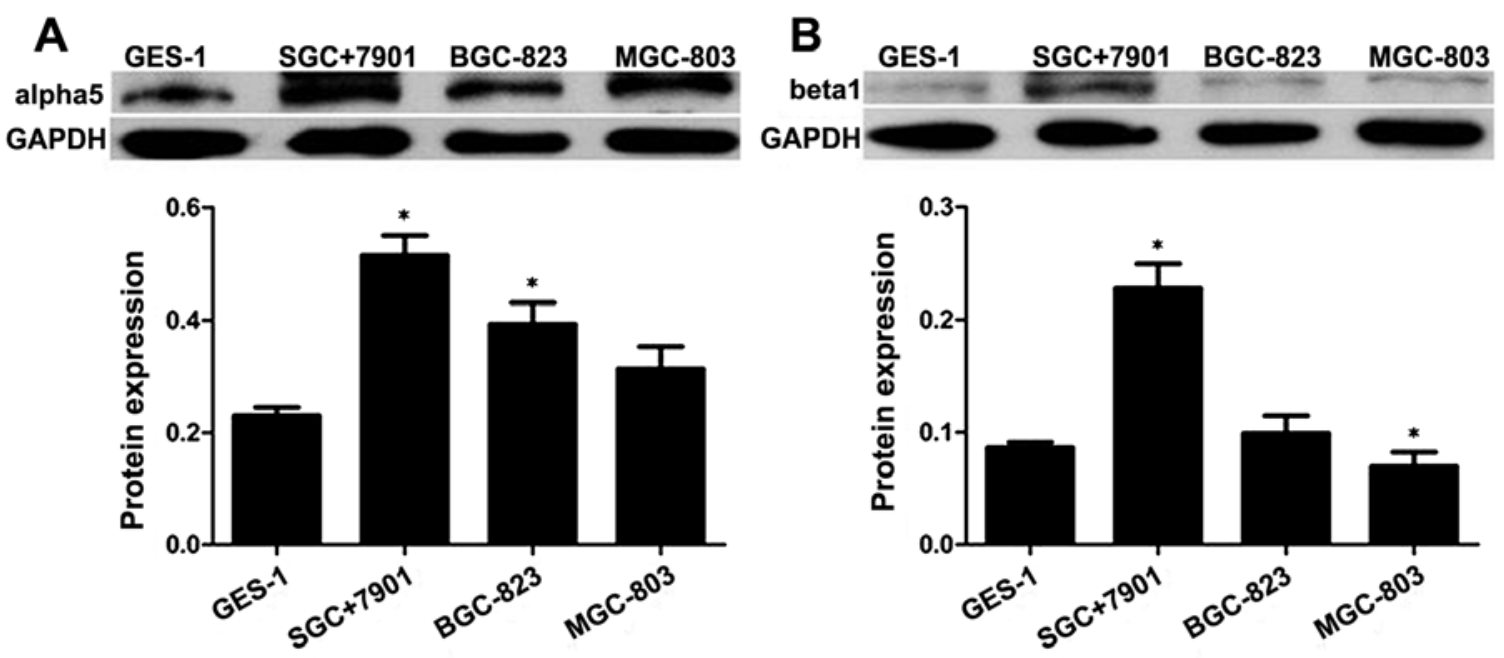

Figure 4. Western blot analysis of (A) integrin $\alpha_{5}$ and (B) $\beta_{1}$ subunits in human GES1, SGC7901, BGC823 and MGC803 cell lines. "P<0.05 (compared with GES1 cells).

Figs. 5 and 6A, the cell-associated fluorescence of RGD-PLS by overexpression of integrin $\alpha_{5} \beta_{1}$ in the SGC7901 and BGC 823 cells was higher than PLS at $37^{\circ} \mathrm{C}$; however, GES1 and MGC803 cells treated with RGD-PLS and PLS had no obvious difference in binding fluorescence. For the SGC7901 and BGC823 cells, the cellular fluorescence intensities of the RGD-PLS cells were as much as 2.26 -fold $(\mathrm{P}<0.05)$ and 1.67-fold $(\mathrm{P}<0.05)$ that of PLS at $37^{\circ} \mathrm{C}$ (Fig. 6B), respectively.

To confirm the specificity of RGD-PLS towards SGC7901 and BGC823 cells, GES1 cells were chosen as the negative control. As presented in Figs. 5 and 6B, there was no significant difference in binding to GES1 cells among the two liposome formulations as expected. The different cell association of RGD-PLS in the different cells indicated that specific cell binding and cellular uptake were dependent on the cell surface expression levels of integrin $\alpha_{5} \beta_{1}$.

Confocal microscopic analysis of cell binding and cellular uptake. In order to investigate the interactions between RGD-PLS and target cells, confocal microscopy was employed to evaluate the cell uptake of coumarin-6-loaded liposomes by SGC7901 and GES1 cells, and non-active targeted liposomes PLS were chosen as the negative controls.

Fig. 7 shows the fluorescence images of SGC7901 and GES1 cells treated with liposomes after a 1-h incubation at $37^{\circ} \mathrm{C}$. For the target SGC7901 cells, some non-specific cell binding was observed with PLS. The fluorescence intensity of the RGD-PLS binding to SGC7901 cells was significantly greater than the intensity of the PLS. For the GES1 cells, we also observed non-specific cell binding treated with PLS, and the fluorescence signal of PLS and RGD-PLS were as weak as the SGC7901 cells with PLS. This suggests that cells had non-specific uptake of the liposomes and RGD markedly improved the specific cell binding and cellular uptake of liposomes by SGC7901 cells.

In vivo imaging in the tumor model. In order to test the active targeting efficiency of the liposomes toward gastric cancer, we established a nude mouse tumor model. Fig. 8A represents the real-time distribution and tumor accumulation 

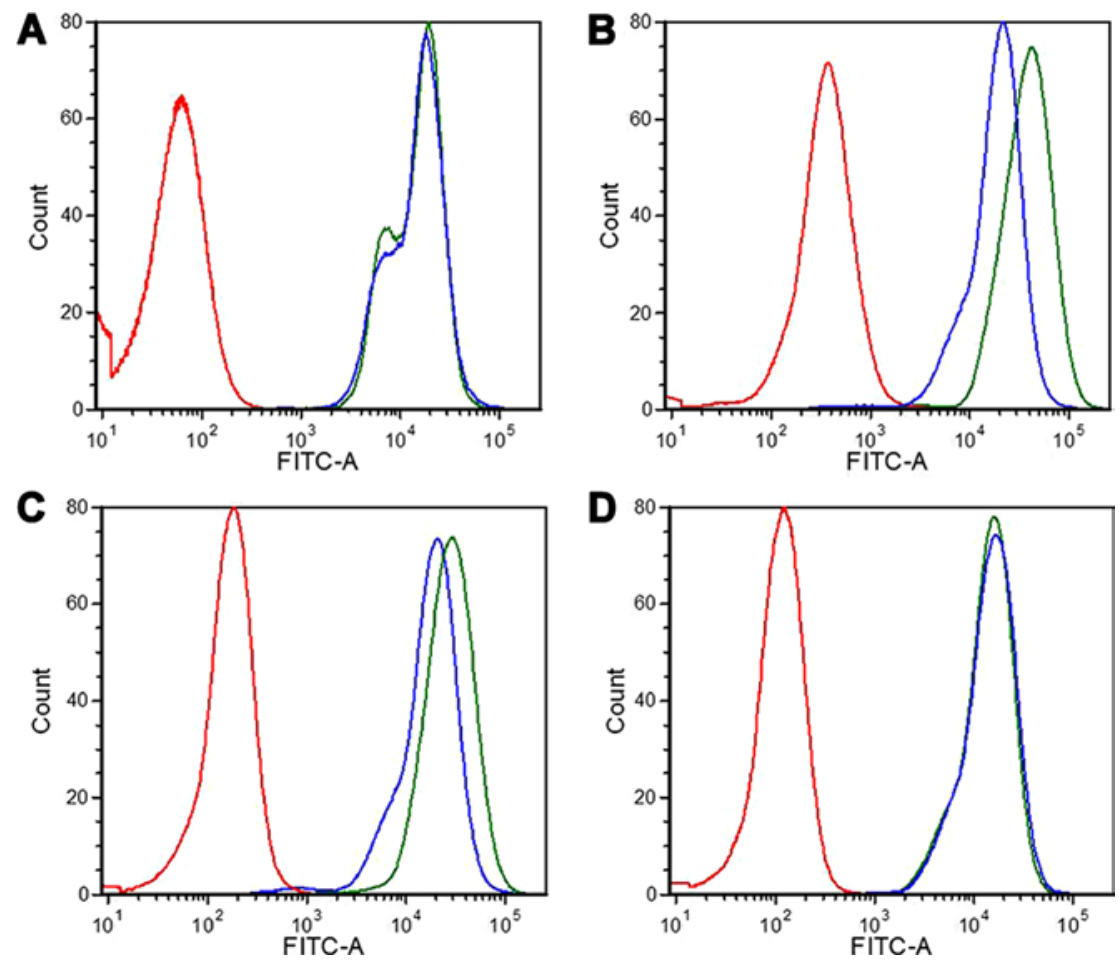

Figure 5. Flow cytometric analysis of coumarin-6 fluorescence intensity in (A) GES1, (B) SGC7901, (C) BGC803 and (D) MGC803 cells. Cells were treated with PLS (blue line) and RGD-PLS (green line) (containing $2 \mu \mathrm{g} / \mathrm{ml}$ coumarin-6) at $37^{\circ} \mathrm{C}$ for $1 \mathrm{~h}$. The GES1 cells were chosen as the blank controls (red line). PLS, PEGylated liposomes.

A

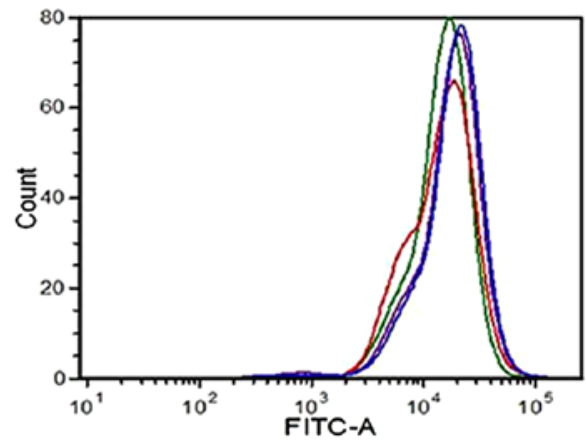

B
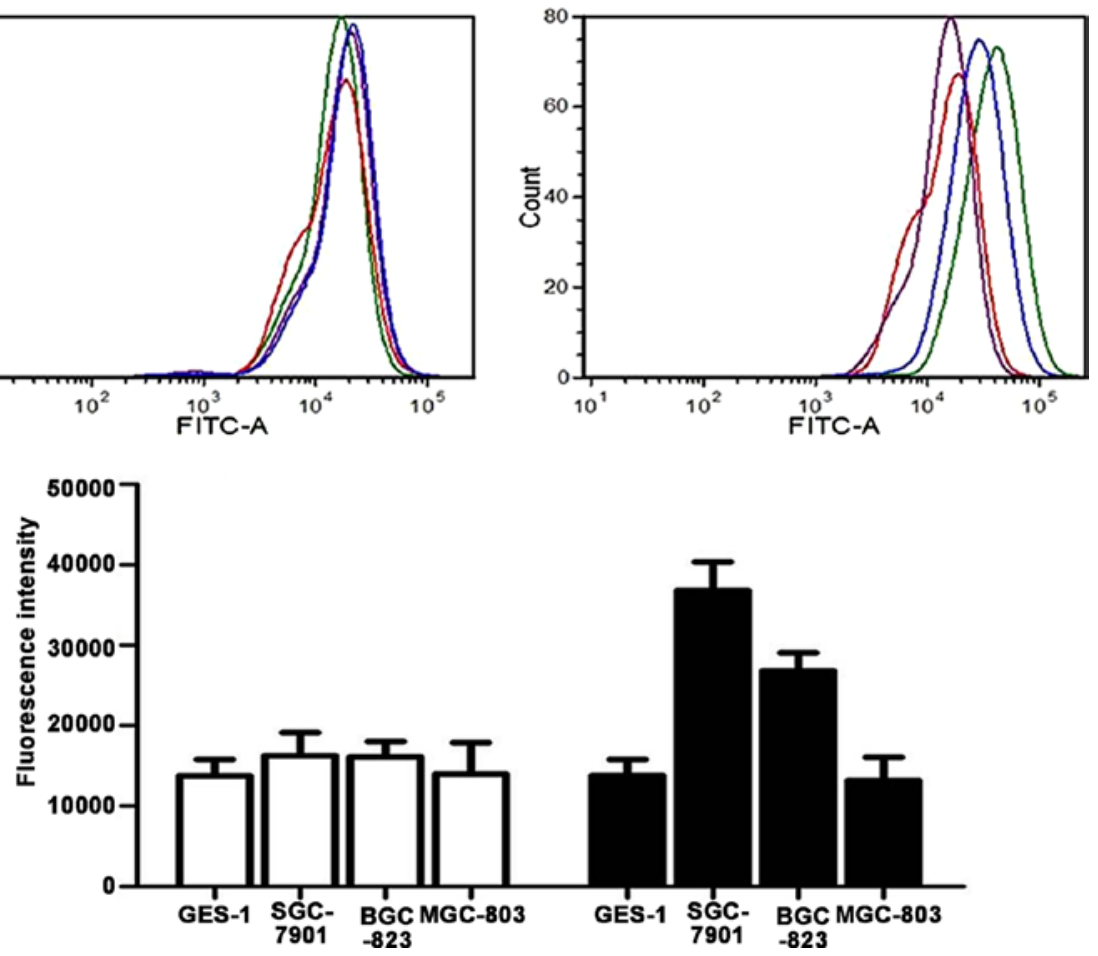

Figure 6. (A) Flow cytometric analysis of coumarin-6 fluorescence intensity in GES1 (red line), SGC7901 (blue line), BGC823 (green line) and MGC803 (purple line) cells, and (B) histogram of flourescence intensity. Cells were incubated with coumarin- 6 loaded PLS and RGD-PLS for $1 \mathrm{~h}$ at $37^{\circ} \mathrm{C}$. "P< $<0.05$ (compared with GES1 cells). PLS, PEGylated liposomes.

ability of the fluorescence ICG-loaded liposomes in the tumor model by intravenous injection into the tail. The fluorescence accumulation was found in tumors of the RGD-PLS groups starting at $16 \mathrm{~h}$ post injection, while limited fluorescence was

$$
\text { (1) }
$$

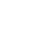


A

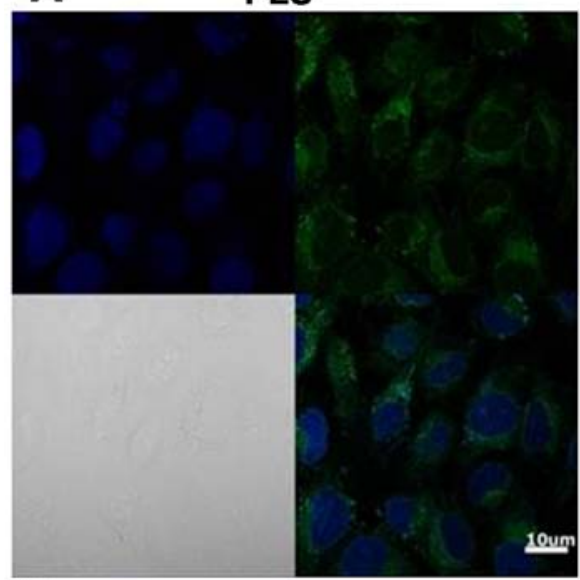

B

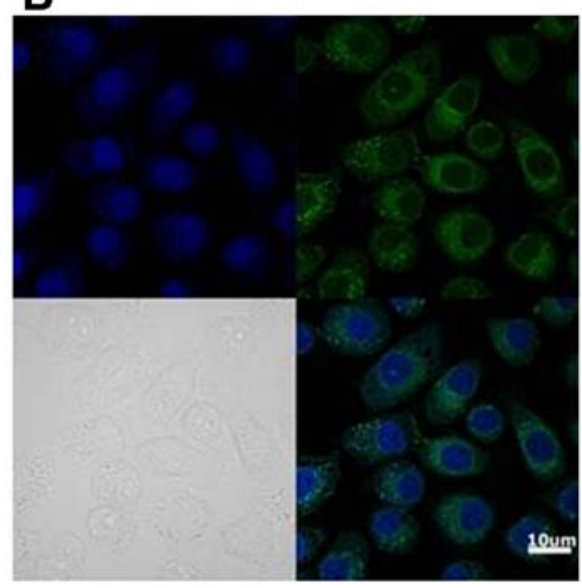

RGD-PLS
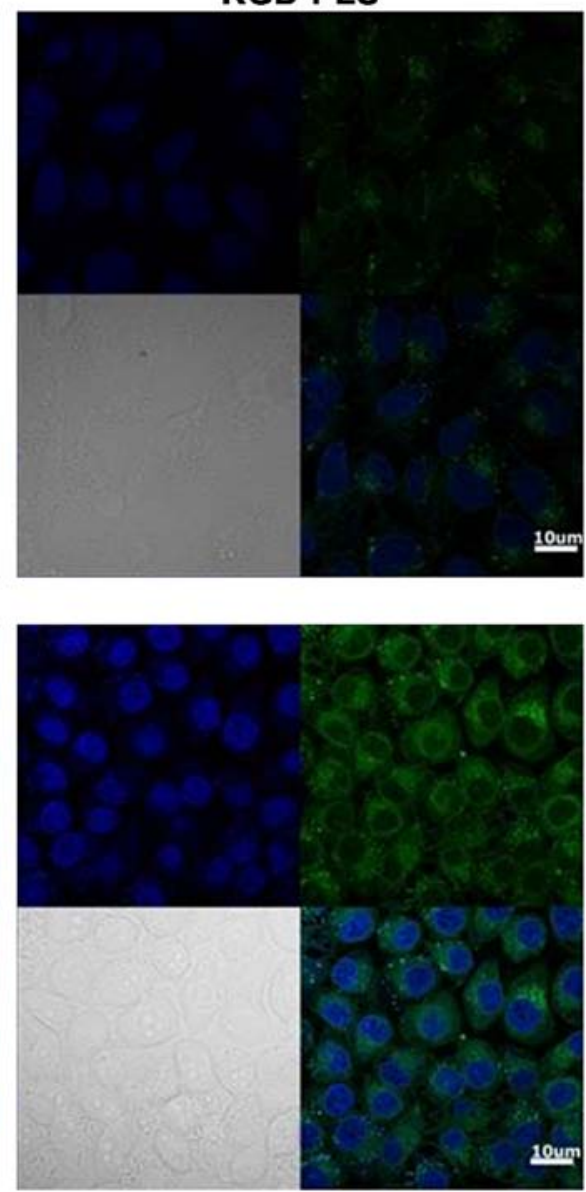

Figure 7. Confocal microscopy images of integrin $\alpha_{5} \beta_{1}$ overexpression in SGC7901 cells and expression in the normal control (GES1) cells following treatment with liposomal formulations. (A) GES1 and (B) SGC7901 cells were incubated with PLS and RGD-PLS with $2 \mu \mathrm{g} / \mathrm{ml}$ coumarin-6 in serum-free medium for $1 \mathrm{~h}$ at $37^{\circ} \mathrm{C}$, respectively. Cell nuclei were stained blue with Hoechst 33342 . Coumarin-6 is shown as green fluorescence. PLS, PEGylated liposomes.

at the same time point. After $120 \mathrm{~h}$, the ICG accumulation in tumors was the largest and still higher than those of PLS. The ex vivo image of excised organs (Fig. 8B) confirmed the higher fluorescence accumulation in the tumors of the RGD-PLS group compare with those of the PLS group. The ICG accumulation in tumors of the PLS group showed that the liposomes can be trapped and retained for longer time periods through the process called enhanced permeation and retention (EPR) effect. However, ICG accumulation of RGD-PLS in the tumors did not only depend on the EPR effect, yet also relied on active targeting of RGD. These results are further proof of the selective accumulation of RGD-PLS in gastric cancer. An additional explanation for the high fluorescence intensity observed in the liver and spleen is the naturally high uptake of liposomes by the macrophages in these organs (Fig. 8B).

\section{Discussion}

In the present study, a novel NIR contrast system containing ICG was developed and its targeting efficiency to gastric cancer in a tumor model was investigated. RGD-PLS-ICG had several advantages over ICG or PLS-ICG, including: i) increased fluorescence signal with a shift toward the longer wavelength absorption; ii) vastly improved stability in 50\% FBS solu- tion; iii) specificity combined with the cells; iv) prolonged circulation time in vivo; v) effective reduction RES uptake; and vi) improved visualization of tumor tissues in vivo. These characteristics of RGD-LP-ICG have enabled quantitative development of tumor tissues in the tumor model.

Fibronectin (FN) is a high-molecule glycoprotein of the ECM that binds to membrane-spanning receptor proteins called integrins, and play an important role in cell adhesion, growth, migration and differentiation. It was previously reported that the majority of integrin-mediated interactions with FN occur through the arg-gly-asp (RGD) cell-binding sequence in repeat $\mathrm{III}_{10}$, such as integrin $\alpha_{5} \beta_{1}, \alpha_{\mathrm{v}} \beta_{3}$ and $\alpha_{\mathrm{IIb}} \beta_{3}(6,7)$. In recent years, integrin $\alpha_{5} \beta_{1}$ was found to be overexpressed in several types of cancers $(35,36)$. Furthermore, substantial evidence suggests that RGD binds to gastric cancer $(15,16)$. This research suggests that RGD may be a promising target ligand for gastric cancer. However, previous studies have mainly focused on RGD as a targeting ligand for the treatment of tumors and limited research has reported its use for tracing tumors. In the present study, we explored expression of integrin $\alpha_{5} \beta_{1}$ in 3 gastric cancer cell lines and normal gastric mucosa epithelial cells. The results showed that integrin $\alpha_{5} \beta_{1}$ had higher expression in the SGC7901 cells than that in the other cell lines. Therefore, we prepared RGD-PLS-ICG to 


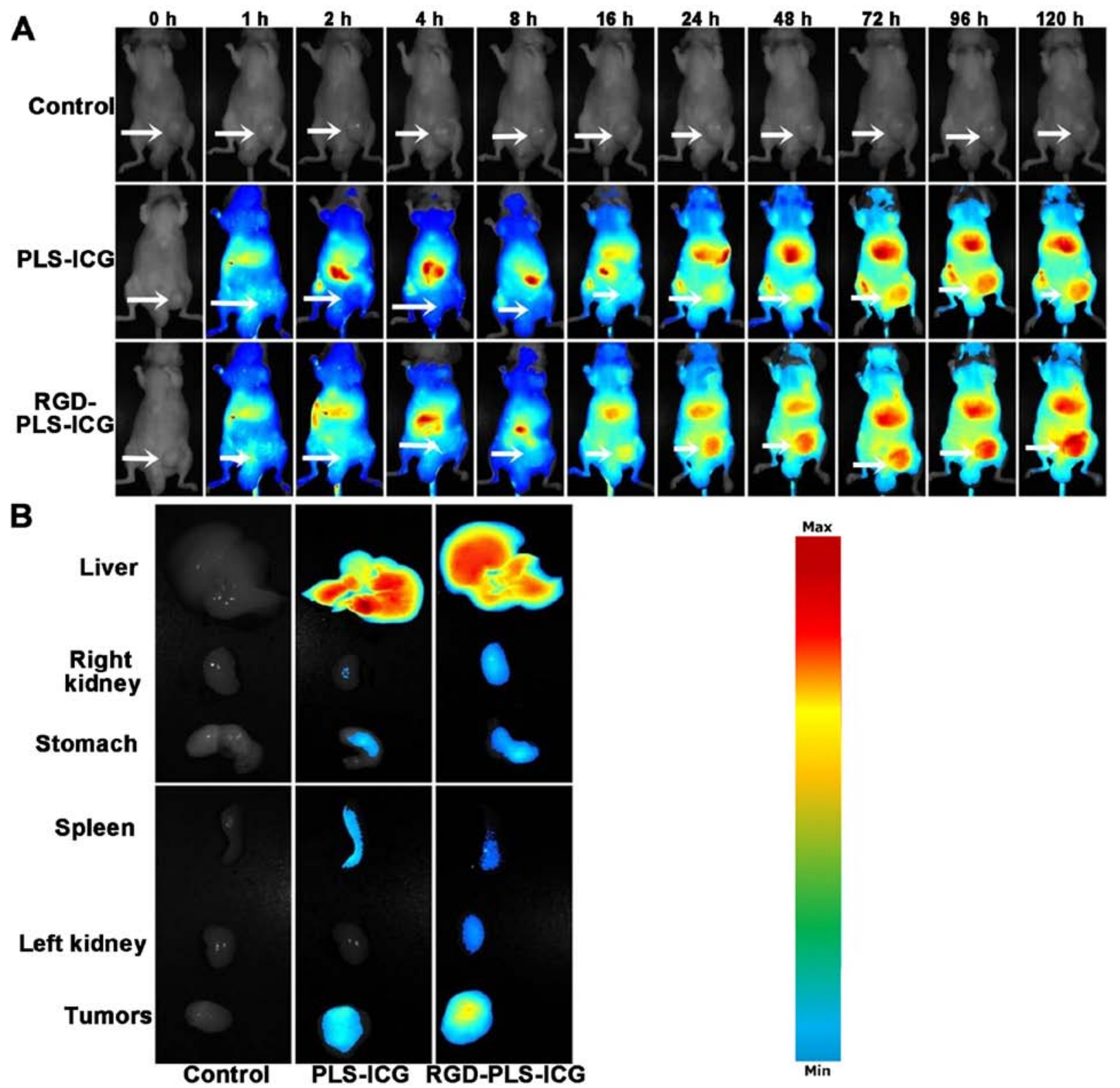

Figure 8. Biodistribution analysis. (A) In vivo NIRF imaging of SGC7901 tumor-bearing mice after intravenous injection of physiological saline, ICG-loaded PLS and ICG-loaded RGD-PLS, respectively. The white arrow indicates the location of the tumor. (B) The ex vivo optical images of organs and tumors of the SGC7901 tumor-bearing mice sacrificed at $120 \mathrm{~h}$ after administration. NIRF, NIR fluorescent; ICG, indocyanine green; PLS, PEGylated liposomes.

confirm that RGD enhanced the gastric cancer targeting effect by increasing specific binding to gastric cancer cells.

ICG is considered one of the most attractive exogenous contrast agents for in vivo NIR fluorescent (NIRF) imaging, due to its spectral properties, minimal toxicity, low cost and FDA-approved status as a medical diagnostic compound (39). Injected ICG is rapidly cleared through the liver and bile duct (40) and it is unstable, aggregates in solution, self-quenches and has low quantum properties (41). These disadvantages are overcome by combining ICG with colloidal particles, such as micelles and liposomes (42). The design of an efficient ICG delivery system preserves its optical properties and prolongs the half-life. For the current application, the optimized formulation exhibited an increased absorbance and a bathochromic shift in the absorption spectrum. The spectral properties of RGD-PLS-ICG and PLS-ICG were preserved in 50\% FBS solution for one week.
In the present experiment, the conjugation of RGD onto the surface of PEGylated liposomes was carried out by DMT-MM as a catalyst, which created stable amide bonds between the amine groups of the PEG chains and the carboxyl groups of RGD in the water phase (28). The particle size of the liposomes plays an important role for the pharmacokinetics. Liposomes with particle size $<200 \mathrm{~nm}$ increase drug accumulation in the tumor via the EPR effect (43). Meanwhile, the particle size of liposomes also has an effect on their interactions with target cells. Particles up to $100-200 \mathrm{~nm}$ can be internalized by receptor-mediated endocytosis (44). In the present study, the sizes of PLS-ICG and RGD-PLS-ICG were mainly 100-130 nm, which would be favorable for passive and active targeting and for cellular uptake.

To demonstrate the specific cell binding and internalization of RGD-PLS, SGC7901, BGC803 and MGC803 cells were chosen as experimental groups, while GES1 cells were applied 
as a control group. Non-active targeted liposomes, PLS, was also prepared and used as the negative controls. The flow cytometric data showed that the cell associated fluorescence of RGD-PLS in SGC7901 and BGC823 cells was higher than that of PLS, while MGC803 cells treated with RGD-PLS and PLS had no obvious differences in the GES1 cells. Receptormediated binding was proceeded at both $4^{\circ} \mathrm{C}$ and $37^{\circ} \mathrm{C}$, yet endocytosis and internalization occurred only at $37^{\circ} \mathrm{C}(45)$. Therefore, we explored how SGC7901, BGC823, MGC803 and GES1 cells may incubate with RGD-PLS and PLS at $37^{\circ} \mathrm{C}$. In the SGC7901 and BGC823 cells, except the non-specific binding event, an additional specific cell binding named the receptor-mediated binding also occurs for RGD-PLS, which was different from PLS (non-specific binding only) (45). In addition, the markedly enhanced uptake of RGD-PLS compared with PLS by SGC7901 and BGC823 cells suggested an easier endocytosis may results from the presence of specific binding mediated by RGD.

Meanwhile, confocal microscopy images demonstrated that RGD-PLS resulted in a significant higher cell association by SGC7901 cells compared to PLS. However, similar cellular behavior was found in the two liposomal formulations when they were incubated in the GES1 cells. All of these phenomena suggested that RGD-PLS enhanced the specific cell binding and cellular uptake in the SGC7901 cells due to RGD, and also depending on the integrin $\alpha_{5} \beta_{1}$ expression level at the cell surface.

One of the most important concerns of a new active targeting delivery system is its tolerability and toxicity upon the interaction with the target and normal cells. PEGylated liposomes, which have been approved by the FDA, are regarded as promising drug carriers with good biocompatibility, biodegradability and low cytotoxicity for cancer therapeutics (46). In addition, RGD has been used in the clinic $(15,16)$. In the present study, we did not explore RGD for cell toxicity in vitro, yet no obvious side-effect was noted during the period of the animal experiments. However, long-term in vivo toxicity and immunogenicity of RGD-PLS in animals should be investigated further.

A biodistribution study was also conducted to investigate the tumor targeting effect of RGD-PLS in vivo. The biodistribution of PLS and RGD-PLS indicated that both passive and active tumor targeting mechanisms were involved in the ICG accumulation within the tumor, in which passive targeting was attributed to the EPR effect of PLS and active targeting may be achieved by RGD conjugation to PLS. As shown in Fig. 8, the accumulation of ICG in tumors was significantly increased after the attachment of RGD to PLS. These results suggest that RGD modification of PEGylated liposomes facilitates the accumulation of PLS in gastric cancer, and the gastric cancer targeting ability of RGD was proven. However, a higher fluorescence intensity was observed in the liver and spleen due to the RES uptake nature for liposomes; how to reduce or to avoid RES uptake is a topic for future research. Based on the present study, RGD-PLS may be a promising delivery system to target gastric cancer with favorable integrin $\alpha_{5} \beta_{1}$ targeting ability. However, future study will be aimed at reducing the uptake of liposomes in the liver and spleen and at finding more specific targeting ligands to gastric cancer.
In conclusion, a gastric cancer targeting fluorescent dye delivery system was successfully constructed by the DMTMM-mediated conjugation of PEGylated liposomes with RGD, where RGD was used for active targeting to gastric cancer and the PEG-lipid was applied for prolonging circulation time in vivo. The coumarin-6 and ICG-loaded RGD-PLS had a particle size of $<200 \mathrm{~nm}$, which would be favorable for passive and active tumor targeting. RGD-PLS was demonstrated to be a promising active targeting tracer delivery system for targeting gastric cancer cells overexpressing integrin, as it possessed marked binding affinity and specificity towards gastric cancer cells, and improved accumulation in stomach tumors. This targeting delivery carrier may be applied in the clinical and may facilitate accurate resection of gastric cancers in the future.

\section{Acknowledgements}

The present study was supported by the National Nature Science Foundation of China (81372364), the Digestive System Disease Clinical Medical Center of Jiangsu Province (BK2012001), and the Nature Science Foundation of Nanjing (ZKX11015).

\section{References}

1. Ferlay J, Shin HR, Bray F, Forman D, Mathers C and Parkin DM: Estimates of worldwide burden of cancer in 2008: GLOBOCAN 2008. Int J Cancer 127: 2893-2917, 2010.

2. Dikken JL, van de Velde CJ, Coit DG, Shah MA, Verheij M and Cats A: Treatment of resectable gastric cancer. Therap Adv Gastroenterol 5: 49-69, 2012.

3. Ajani JA, Barthel JS, Bekaii-Saab T, Bentrem DJ, D'Amico TA, Das P, Denlinger C, Fuchs CS, Gerdes H, Hayman JA, et al; NCCN Gastric Cancer Panel: Gastric cancer. J Natl Compr Canc Netw 8: 378-409, 2010.

4. Okines A, Verheij M, Allum W, Cunningham D and Cervantes A; ESMO Guidelines Working Group: Gastric cancer: ESMO Clinical Practice Guidelines for diagnosis, treatment and followup. Ann Oncol 21 (Suppl 5): v50-v54, 2010.

5. Songun I, Putter H, Kranenbarg EMK, Sasako M and van de Velde CJH: Surgical treatment of gastric cancer: 15 -year follow-up results of the randomised nationwide Dutch D1D2 trial. Lancet Oncol 11: 439-449, 2010.

6. Ruoslahti E: Integrins. J Clin Invest 87: 1-5, 1991.

7. Hynes RO: Integrins: Versatility, modulation, and signaling in cell adhesion. Cell 69: 11-25, 1992.

8. Aota S, Nomizu M and Yamada KM: The short amino acid sequence Pro-His-Ser-Arg-Asn in human fibronectin enhances cell-adhesive function. J Biol Chem 269: 24756-24761, 1994.

9. Rajeswari J and Pande G: The significance of alpha 5 beta 1 integrin dependent and independent actin cytoskelton organization in cell transformation and survival. Cell Biol Int 26: 1043-1055, 2002

10. Toquet C, Colson A, Jarry A, Bezieau S, Volteau C, Boisseau P, Merlin D, Laboisse CL and Mosnier JF: ADAM15 to $\alpha 5 \beta 1$ integrin switch in colon carcinoma cells: A late event in cancer progression associated with tumor dedifferentiation and poor prognosis. Int J Cancer 130: 278-287, 2012.

11. Nam JM, Onodera Y, Bissell MJ and Park CC: Breast cancer cells in three-dimensional culture display an enhanced radioresponse after coordinate targeting of integrin alpha5betal and fibronectin. Cancer Res 70: 5238-5248, 2010.

12. Mitra AK, Sawada K, Tiwari P, Mui K, Gwin K and Lengyel E: Ligand-independent activation of c-Met by fibronectin and $\alpha_{5} \beta_{1}$-integrin regulates ovarian cancer invasion and metastasis. Oncogene 30: 1566-1576, 2011.

13. Chi F, Fu D, Zhang X, Lv Z and Wang Z: Expression of the c-Met proto-oncogene and Integrin $\alpha 5 \beta 1$ in human gastric cardia adenocarcinoma. Biosci Biotechnol Biochem 76: 1471-1476, 2012. 
14. Ren J, Xu S, Guo D, Zhang J and Liu S: Increased expression of $\alpha 5 \beta 1$-integrin is a prognostic marker for patients with gastric cancer. Clin Transl Oncol 16: 668-674, 2014.

15. Chen CH, Liu DZ, Fang HW, Liang HJ, Yang TS and Lin SY: Evaluation of multi-target and single-target liposomal drugs for the treatment of gastric cancer. Biosci Biotechnol Biochem 72: 1586-1594, 2008.

16. Wang C, Bao C, Liang S, Fu H, Wang K, Deng M, Liao Q and Cui D: RGD-conjugated silica-coated gold nanorods on the surface of carbon nanotubes for targeted photoacoustic imaging of gastric cancer. Nanoscale Res Lett 9: 264, 2014.

17. Kusano M, Tajima Y, Yamazaki K, Kato M, Watanabe M and Miwa M: Sentinel node mapping guided by indocyanine green fluorescence imaging: A new method for sentinel node navigation surgery in gastrointestinal cancer. Dig Surg 25: 103-108, 2008.

18. Tajima Y, Murakami M, Yamazaki K, Masuda Y, Kato M, Sato A, Goto S, Otsuka K, Kato T and Kusano M: Sentinel node mapping guided by indocyanine green fluorescence imaging during laparoscopic surgery in gastric cancer. Ann Surg Oncol 17: 1787-1793, 2010.

19. Bangham AD and Horne RW: Negative staining of phospholipids and their structural modification by surface-active agents as observed in the electron microscope. J Mol Biol 8: 660-668, 1964.

20. Anders CK, Adamo B, Karginova O, Deal AM, Rawal S, Darr D, Schorzman A, Santos C, Bash R, Kafri T, et al: Pharmacokinetics and efficacy of PEGylated liposomal doxorubicin in an intracranial model of breast cancer. PLoS One 8: e61359, 2013.

21. Lin YY, Kao HW, Li JJ, Hwang JJ, Tseng YL, Lin WJ, Lin MH, Ting $\mathrm{G}$ and Wang HE: Tumor burden talks in cancer treatment with PEGylated liposomal drugs. PLoS One 8: e63078, 2013.

22. Jiang J, Yang SJ, Wang JC, Yang LJ, Xu ZZ, Yang T, Liu XY and Zhang Q: Sequential treatment of drug-resistant tumors with RGD-modified liposomes containing siRNA or doxorubicin. Eur J Pharm Biopharm 76: 170-178, 2010.

23. Yamamoto Y, Yoshida M, Sato M, Sato K, Kikuchi S, Sugishita H, Kuwabara J, Matsuno Y, Kojima Y, Morimoto M, et al: Feasibility of tailored, selective and effective anticancer chemotherapy by direct injection of docetaxel-loaded immunoliposomes into Her2/neu positive gastric tumor xenografts. Int J Oncol 38: 33-39, 2011.

24. Sandanaraj BS, Gremlich HU, Kneuer R, Dawson J and Wacha S: Fluorescent nanoprobes as a biomarker for increased vascular permeability: Implications in diagnosis and treatment of cancer and inflammation. Bioconjug Chem 21: 93-101, 2010.

25. Proulx ST, Luciani P, Derzsi S, Rinderknecht M, Mumprecht V, Leroux JC and Detmar M: Quantitative imaging of lymphatic function with liposomal indocyanine green. Cancer Res 70: 7053-7062, 2010 .

26. Duncan R and Sat YN: Tumour targeting by enhanced permeability and retention (EPR) effect. Ann Oncol 9 (Suppl 2): 39-39, abs. 149, 1998.

27. Hope MJ, Bally MB, Webb G and Cullis PR: Production of large unilamellar vesicles by a rapid extrusion procedure: Characterization of size distribution, trapped volume and ability to maintain a membrane potential. Biochim Biophys Acta 812: 55-65, 1985

28. Kunishima M, Kawachi C, Hioki K, Terao R and Tani S: Formation of carboxamides by direct condensation of carboxylic acids and amines in alcohols using a new alcohol-and watersoluble condensing agent: DMT-MM. Tetrahedron 57: 1551-1558, 2001.
29. Jeong HS, Lee CM, Cheong S-J, Kim EM, Hwang H, Na KS Lim ST, Sohn MH and Jeong HJ: The effect of mannosylation of liposome-encapsulated indocyanine green on imaging of sentinel lymph node. J Liposome Res 23: 291-297, 2013.

30. Livak KJ and Schmittgen TD: Analysis of relative gene expression data using real-time quantitative PCR and the $2^{-\Delta \Delta C T}$ method. Methods 25: 402-408, 2001.

31. Kao E, Shinohara M, Feng M, Lau MY and Ji C: Human immunodeficiency virus protease inhibitors modulate $\mathrm{Ca}^{2+}$ homeostasis and potentiate alcoholic stress and injury in mice and primary mouse and human hepatocytes. Hepatology 56: 594-604, 2012.

32. Simard P and Leroux JC: pH-sensitive immunoliposomes specific to the CD33 cell surface antigen of leukemic cells. Int J Pharm 381: 86-96, 2009.

33. Mordon S, Devoisselle JM, Soulie-Begu S and Desmettre T: Indocyanine green: Physicochemical factors affecting its fluorescence in vivo. Microvasc Res 55: 146-152, 1998.

34. Desmettre T, Devoisselle JM, Soulie-Begu S and Mordon S: Fluorescence properties and metabolic features of indocyanine green (ICG). J Fr Ophtalmol 22: 1003-1016, 1999 (In French).

35. Gulubova M and Vlaykova T: Immunohistochemical assessment of fibronectin and tenascin and their integrin receptors alpha5beta 1 and alpha9beta1 in gastric and colorectal cancers with lymph node and liver metastases. Acta Histochem 108: 25-35, 2006.

36. Albrecht $\mathrm{M}$, Renneberg $\mathrm{H}$, Wennemuth $\mathrm{G}$, Möschler $\mathrm{O}$, Janssen M, Aumüller G and Konrad L: Fibronectin in human prostatic cells in vivo and in vitro: Expression, distribution, and pathological significance. Histochem Cell Biol 112: 51-61, 1999.

37. Kesanakurti D, Chetty C, Dinh DH, Gujrati M and Rao JS: Role of MMP-2 in the regulation of IL-6/Stat3 survival signaling via interaction with $\alpha 5 \beta 1$ integrin in glioma. Oncogene 32: 327-340, 2013.

38. Gahmberg CG, Fagerholm SC, Nurmi SM, Chavakis T, Marchesan S and Grönholm M: Regulation of integrin activity and signalling. Biochim Biophys Acta 1790: 431-444, 2009.

39. Frangioni JV: In vivo near-infrared fluorescence imaging. Curr Opin Chem Biol 7: 626-634, 2003.

40. Unno N, Nishiyama M, Suzuki M, Yamamoto N, Inuzuka K, Sagara D, Tanaka H and Konno H: Quantitative lymph imaging for assessment of lymph function using indocyanine green fluorescence lymphography. Eur J Vasc Endovasc Surg 36: 230-236, 2008.

41. Saxena V, Sadoqi M and Shao J: Indocyanine green-loaded biodegradable nanoparticles: Preparation, physicochemical characterization and in vitro release. Int J Pharm 278: 293-301, 2004.

42. Ohnishi S, Lomnes SJ, Laurence RG, Gogbashian A, Mariani G and Frangioni JV: Organic alternatives to quantum dots for intraoperative near-infrared fluorescent sentinel lymph node mapping. Mol Imaging 4: 172-181, 2005.

43. Gao J, Zhong W, He J, Li H, Zhang H, Zhou G, Li B, Lu Y, Zou H, Kou G, et al: Tumor-targeted PE38KDEL delivery via PEGylated anti-HER2 immunoliposomes. Int J Pharm 374: 145-152, 2009.

44. Couvreur P and Puisieux F: Nanoparticles and microparticles for the delivery of polypeptides and proteins. Adv Drug Deliv Rev 10: 141-162, 1993.

45. Xie H, Zhu Y, Jiang W, Zhou Q, Yang H, Gu N, Zhang Y, Xu H, $\mathrm{Xu} \mathrm{H}$ and Yang $\mathrm{X}$ : Lactoferrin-conjugated superparamagnetic iron oxide nanoparticles as a specific MRI contrast agent for detection of brain glioma in vivo. Biomaterials 32: 495-502, 2011.

46. Allen TM and Cullis PR: Drug delivery systems: Entering the mainstream. Science 303: 1818-1822, 2004. 